\title{
Urinary capillariosis in six dogs from Italy
}

\author{
A. Mariacher $1,2, *$, F. Millanta ${ }^{2}$, G. Guidi² and S. Perrucci ${ }^{2}$ \\ ${ }^{1}$ Istituto Zooprofilattico Sperimentale delle Regioni Lazio e Toscana, Viale Europa 30, 58100 Grosseto, Italy \\ ${ }^{2}$ Dipartimento di Scienze Veterinarie, Viale delle Piagge 2, 56124 Pisa, Italy
}

\begin{abstract}
Canine urinary capillariosis is caused by the nematode Pearsonema plica. P. plica infection is seldomly detected in clinical practice mainly due to diagnostic limitations. This report describes six cases of urinary capillariosis in dogs from Italy. Recurrent cystitis was observed in one dog, whereas another patient was affected by glomerular amyloidosis. In the remaining animals, the infection was considered an incidental finding. Immature eggs of the parasite were observed with urine sediment examination in 3/6 patients. Increased awareness of the potential pathogenic role of P. plica. and clinical disease presentation could help identify infected animals.
\end{abstract}

Keywords: Cystitis, Dog, Glomerular amyloidosis, Urinary capillariosis.

\section{Introduction}

Urinary capillariosis in dogs is caused by Pearsonema plica (Trichurida, Capillariidae), a nematode that infects domestic and wild carnivores worldwide. Clinical cases of canine urinary capillariosis have been reported in the United States (Senior et al., 1980; Kirkpatrick and Nelson, 1987) and Europe, including France (Cazelles et al., 1989), Switzerland (Spillmann and Glardon, 1989; Basso et al., 2014), Holland (van Veen, 2002), Poland (Studzińska et al., 2015) and Italy (Callegari et al., 2010; Maurelli et al., 2014). Adult parasites live superficially attached to the mucosa of the urinary bladder, while the ureters and renal pelvis are seldom affected (Bork-Mimm and Rinder, 2011).

$P$. plica has an indirect life cycle that involves earthworms (Lumbricina) as intermediate hosts (Senior et al., 1980) and domestic and wild carnivores as definitive hosts (Anderson, 2000). P. plica is most often reported in dogs with access to outdoor environments, especially hunting dogs and kenneled dogs (Senior et al., 1980; Callegari et al., 2010). The red fox (Vulpes vulpes) is considered the wild reservoir of P. plica for domestic animals in Europe (Davidson et al., 2006; Magi et al., 2014), but the wolf (Canis lupus) is emerging as an additional reservoir (Bagrade et al., 2009; Mariacher et al., 2015). Despite the high prevalence in the red fox, urinary capillariosis has been rarely reported in dogs in Italy (Callegari et al., 2010; Maurelli et al., 2014) and the real occurrence of the disease is probably underestimated.

Indeed, urinary capillariosis has often been regarded as having minor clinical and pathological significance, due to frequent subclinical presentations and limitations in its diagnosis (Otranto, 2015). However, in heavy parasite loads, $P$. plica may be responsible for severe lower urinary tract maladies, both in domestic (Rossi et al., 2011; Basso et al., 2014) and wild carnivores (Fernández-Aguilar et al., 2010; Bork-Mimm and Rinder, 2011; Alić et al., 2015). These urinary disorders generally include pollakiuria, dysuria and hematuria, may have chronic or recurrent clinical forms and do not improve after symptomatic or empirical antibiotic treatments (Senior et al., 1980; van Veen, 2002; Rossi et al., 2011).

Diagnosis of $P$. plica infection is based on examination of the urine sediment, an assessment seldom performed in the routine parasite diagnoses of dogs and cats (Otranto, 2015) as it is mostly reserved for symptomatic patients. Moreover, false negative results may commonly occur even when $P$. plica infection is established and symptomatic, due to a long prepatent period, irregular elimination of low numbers of eggs and difficulties in detecting immature or atypical eggs (Senior et al., 1980; Rossi et al., 2011; Basso et al., 2014; Maurelli et al., 2014). This study describes six cases of urinary capillariosis in dogs, and highlights the variability of its clinical presentation.

\section{Case details}

Six cases of urinary capillariosis were examined in two different veterinary facilities in Italy. Diagnoses were based on the detection of mature and immature P. plica eggs in the urinary sediment of the six dogs. Eggs were identified as P. plica based on: 1) mature eggs are barrel-shaped, colorless and operculated, show a slightly pitted wall and bipolar plugs and measure 55-68 $\mu \mathrm{m} \times 24-29 \mu \mathrm{m}$ (Basso et al., 2014); and/or 2) immature eggs are smaller, lack a shell and show rudimentary polar plugs (Basso et al., 2014). Clinical details and findings at urine sediment examination are summarised in Tables 1 and 2 respectively. 
Case 1

Case 1 was an intact male Maremma hound aged five. The dog had access to rural and forest environments during hunting season. The dog was referred for polyuria/polydipsia and was diagnosed with chronic kidney disease at stage III (International Renal Interest Society, 2013) and nephrotic syndrome. Urinary capillariosis was diagnosed based on the presence of mature $P$. plica eggs in the urinary sediment; a slight pyuria was also present. Serological tests were negative for Leishmania sp. and tick-borne diseases, except for a low positive IgG titer (1:40) for Ehrlichia canis. The dog was treated with ramipril (VASOTOP; MSD Animal Health), dietary protein restriction, and fenbendazole $(50 \mathrm{mg} / \mathrm{Kg}$ orally once a day for seven days). Urine sediment examination was repeatedly negative for parasite eggs starting seven days after treatment. Due to worsening proteinuria, a renal biopsy was performed a month later. Histopathology showed glomerular amyloidosis (Fig. 1), chronic interstitial nephritis, and scattered foci of tubular necrosis.

Case 2

Case 2 was an intact male mixed-breed dog aged four. The dog had access to rural and forest environments during hunting season. This patient was referred for leishmaniasis, with no history of urinary signs and renal function parameters were in their normal range. Typical mature eggs of $P$. plica were identified in the urine sediment (Fig. 2), along with a slight pyuria. Urinary capillariosis was treated with fenbendazole (50 $\mathrm{mg} / \mathrm{Kg}$ orally once a day for seven days), and the urine sediment was negative for parasite eggs at seven and fourteen days after treatment.

Case 3

Case 3 was an intact male mixed-breed dog aged four, who was recently rescued from a dog shelter. The dog was presented for an evaluation of leishmaniasis, he had no history of urinary signs and renal function parameters were in their normal range. Mature eggs of P. plica were identified in the urine sediment, along with slight pyuria and hematuria. Urinary capillariosis was treated with fenbendazole $(50 \mathrm{mg} / \mathrm{Kg}$ orally once a day for seven days), and the urine sediment was negative for parasite eggs at seven and fourteen days after treatment.

\section{Case 4}

Case 4 was an intact male mixed-breed dog aged eight, who lived indoors in an urban setting. The patient had a history of recurrent cystitis and hematuria. At physical examination the dog showed gross hematuria, and at urine sediment examination severe hematuria, moderate pyuria and rare immature eggs of $P$. plica were observed. Because only immature eggs were found the infection was not promptly identified in this case, and the dog only received symptomatic treatment 
Table 2. Findings at urine sediment examination in six dogs with urinary capillariosis.

\begin{tabular}{lccccccccc}
\hline Case ID & $S G$ & $p H$ & $P U / C U$ & WBC & RBC & Hyaline casts & Crystals & Bacteria & Epithelial cells \\
\hline Case 1 & 1010 & 6.5 & 9.56 & + & 0 & 0 & 0 & 0 & + \\
Case 2 & 1030 & 8.0 & 0.25 & + & 0 & 0 & 0 & 0 & + \\
Case 3 & 1060 & 7.0 & 0.18 & + & + & 0 & Struvite & 0 & ++ \\
& & & & & & +++ & 0 & 0 & ++ \\
Case 4 & 1050 & 6.0 & n.a. & ++ & ++++ & + & 0 & 0 & + \\
Case 5 & 1060 & 5.0 & n.a. & + & 0 & 0 & Struvite & 0 & + \\
Case 6 & 1030 & 8.0 & n.a. & + & ++++ & 0 & + & + \\
\hline
\end{tabular}

SG: Specific gravity; PU/CU: Protein/creatinine ratio; WBC: White blood cells, RBC: Red blood cells; n.a.: Data not available. Normal ranges for these parameters are as follows: SG: 1030-1040, pH: 5.5-7.0, PU/CU: 0.1-0.5, WBC: 0, RBC: 0, casts: 0, crystals: 0 , bacteria: 0 , epithelial cells: $0 /+$

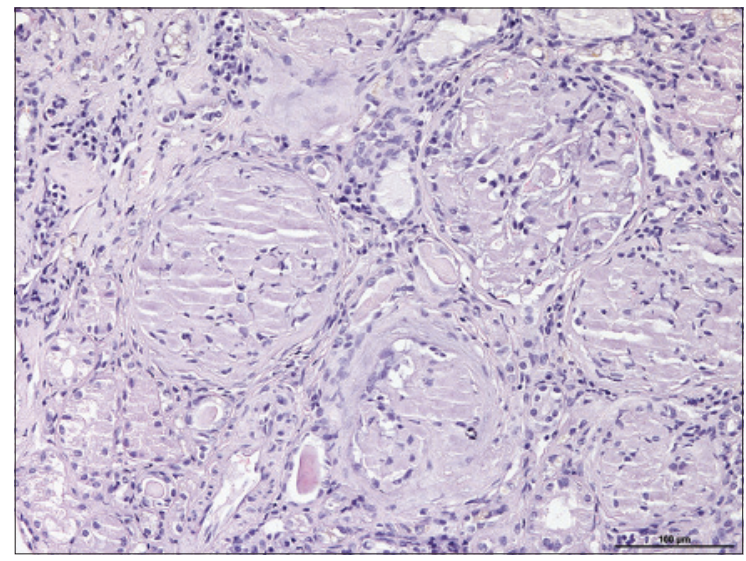

Fig. 1. Canine kidney (case 1): renal glomeruli showing the presence of amorphous hyaline and eosinophilic extracellular material suggestive of amyloid deposition $(\mathrm{H} \& \mathrm{E}, \mathrm{Bar}=100 \mu \mathrm{m})$.

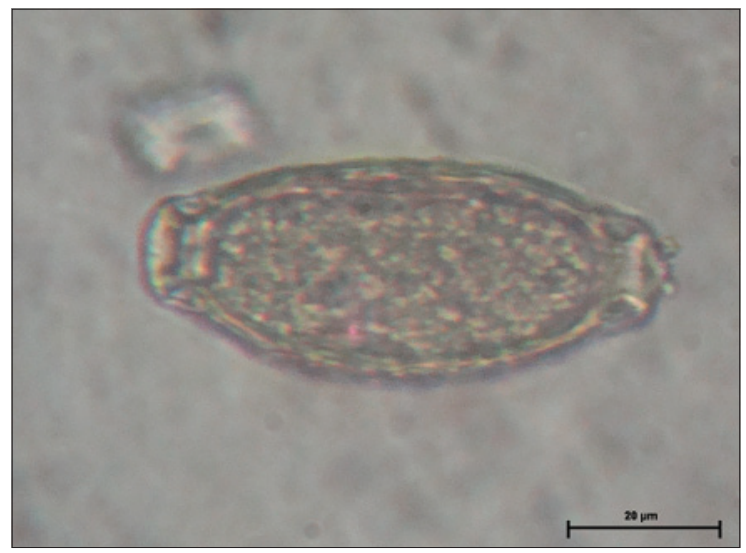

Fig. 2. Urine sediment examination. Pearsonema plica mature egg, showing a thick wall and bipolar plugs (Bar $=$ $20 \mu \mathrm{m})$.

with non-steroidal anti-inflammatory drugs. Six months after the initial examination, the dog was presented for a routine health control and the resulting urine sediment examination was negative for $P$. plica.

\section{Case 5}

Case 5 was an intact male Lhasa Apso aged three, who lived indoors in an urban setting. The dog was presented for lethargy and fever. Immature eggs of $P$. plica were identified in the urine sediment, along with a slight pyuria. Physical examination and clinical laboratory tests did not identify a specific cause of disease and a fever of unknown origin was eventually diagnosed. The dog did not receive any specific treatment for urinary capillariosis and was later lost to follow-up.

\section{Case 6}

Case 6 was a neutered female American Staffordshire aged nine, who lived indoors in an urban setting. The patient was presented with urinary tenesmus. At urine sediment examination severe hematuria, slight pyuria and immature eggs of $P$. plica were observed. A pelvic hemangiosarcoma compressing the urethra was diagnosed with computed tomography and histopathology. Because of the poor prognosis, the owners consented to perform euthanasia.

\section{Discussion}

Of the six cases of urinary capillariosis presented here, one dog showed characteristic clinical signs, consisting of recurrent episodes of hematuria and cystitis (case 4). This patient was asymptomatic six months after the initial diagnosis and its urine sediment was negative for capillariid eggs, despite not receiving any antiparasitic treatment. This finding could be explained by the gradual decrease in the excretion of eggs in the urine, which can occur within a couple of months from the onset of the patent infection (Senior et al., 1980).

The fact that self-limiting infections may occur must not lead to neglecting urinary capillariosis in terms of diagnosis and therapy. There are reports of cases that feature severe clinical signs that may be in relation to a high number of adult parasites and to the chronicity of the infection (Rossi et al., 2011; Basso et al., 2014). Furthermore, it cannot be excluded that urinary 
capillariosis may contribute to the onset or worsening of renal disease.

Indeed, one of the infected animals (case 1) showed considerable similarities to a dog previously described by Callegari et al. (2010) that was affected by glomerular amyloidosis and chronic interstitial nephritis. Since in both cases common infectious diseases were ruled out, $P$. plica infection was considered to be a contributing factor to the deposition of amyloid substance, via a chronic antigenic and inflammatory stimulus (Callegari et al., 2010).

In the remaining four cases, the detection of eggs in the sediment was considered an incidental finding. The absence of clinical signs, in these cases, may be due to a low number of adult parasites in the urinary tract or to their only very superficial attachment to the bladder mucosa (Kruger and Osborne, 1993; Bowman et al., 2002).

In all the cases presented, urine sediment examination showed slight pyuria in absence of bacteria, while hematuria was not a constant finding with 2/6 dogs exhibiting both gross and microscopic hematuria.

In cases 4, 5 and 6, only immature eggs, lacking the outer shell and with rudimentary opercules, were detected in the urine sediment. The regular presence of immature, along with mature, $P$. plica eggs in the urinary sediment of an infected dog has been reported in a previous study (Basso et al., 2014). Data from this study confirm this finding and indicate that in infected dogs the detection of immature $P$. plica eggs may be rather frequent. In this study, two of the three infected dogs in which only immature eggs were isolated, showed no typical signs of urinary capillariosis. This may be due to a low parasite load, or it is possible that finding only immature eggs may be more likely when the number of eggs in the urinary sediment is low. Moreover, this result also indicates that a specific parasitological experience is needed to diagnose the infection in similar cases.

Cases 4, 5 and 6 lived exclusively indoors and were only walked outside on a lead by their owners. This finding suggests that contact with infected intermediate hosts can easily occur even in urban environments. Therefore, P. plica infection should always be included in the differential diagnosis of diseases of the lower urinary tract, even when the patient does not live outdoors. The spread of foxes into urban and periurban areas is a common phenomenon occurring in many European countries (Sréter et al., 2003) and this could result in an increased risk of infection for pets. Two other cases (cases 1 and 2) involved hunting dogs working in rural and forest environments, where they were most likely exposed to settings contaminated by wild reservoir hosts (foxes and wolves). Case 3 was recently rescued from a dog shelter, an environment where infection by $P$. plica is known to be easily maintained and spread (Senior et al., 1980).

Interestingly, 5/6 infected animals were intact males, but sex predilection was not observed in a previous study by Senior et al. (1980) involving a larger number of animals.

Treatment of $P$. plica infection can be challenging and over time it has been attempted with off-label use of different drugs such as: benzimidazoles, avermectines and levamisole. However, to date there are no approved drugs for treating dogs with urinary capillariosis (Senior et al., 1980; Kirkpatrick and Nelson, 1987; van Veen, 2002; Basso et al., 2014; Studzińska et al., 2015). Treatment with fenbendazole performed on three animals in this study appeared to be effective; however, it was recently pointed out that standard urinary sediment examination is not the most adequate way to monitor treatment effectiveness, and more sensitive methods should be used (Otranto, 2015).

In conclusion, data from the present study emphasize the importance of including urinary capillariosis in the differential diagnosis of both chronic and recurrent lower urinary tract and renal disease. Clinicians should be aware that false negative results can be obtained at urinary sediment examination in symptomatic patients, due to low sensitivity of the technique and to parasite biology. Moreover, infection may run asymptomatic, so routine parasitological examination of the urine sediment is advisable in dogs, regardless of the presence or absence of urinary clinical signs. Recently, FLOTAC and Mini-FLOTAC were found to be more sensitive than the classic sedimentation technique in detecting Pearsonema eggs in urine specimens (Maurelli et al., 2014). Furthermore, in infected dogs ultrasonographic examination may reveal hyperechogenicity and thickening of the bladder wall, while endoscopic examination of the urine bladder may detect viable $P$. plica worms attached to the mucosa (Basso et al., 2014).

Development and adoption of more sensitive techniques would be welcome in order to help clinicians diagnose and monitor urinary capillariosis.

\section{Conflict of interest}

The authors declare that there is no conflict of interest.

\section{Acknowledgements}

The authors wish to thank Dr. Francesca Ceccotto and her colleagues at Clinica Veterinaria Serenissima (Sacile, UD; Italy) who provided data for this study.

\section{References}

Alić, A., Hodxić, A., Kadrić, M., Besirović, H. and Prasović, S. 2015. Pearsonema plica (Capillaria plica) infection and associated urinary bladder pathology in red foxes (Vulpes vulpes) from Bosnia and Herzegovina. Parasitol. Res. DOI: 10.1007/ s00436-015-4382-6. 
Anderson, R.C. 2000. Nematode parasites of vertebrates: their development and transmission. CABI Publishing, UK.

Bagrade, G., Kirjušina, M., Vismanis, K. and Ozoliņš, J. 2009. Helminth parasites of the wolf Canis lupus from Latvia. J. Helminthol. 83, 63-68.

Basso, W., Spänhauer, Z., Arnold, S. and Deplazes, P. 2014. Capillaria plica (syn. Pearsonema plica) infection in a dog with chronic pollakiuria: challenges in the diagnosis and treatment. Parasitol. Int. 63, 140-142.

Bork-Mimm, S. and Rinder, H. 2011. High prevalence of Capillaria plica infections in red foxes (Vulpes vulpes) in Southern Germany. Par. Res. 108, 1063-1067.

Bowman, D.D., Hendrix, C.M., Lindsay, D.S. and Barr, S.C. 2002. Feline Clinical Parasitology. Iowa State University Press, Ames, Iowa, USA.

Callegari, D., Kramer, L., Cantoni, A.M., Di Lecce, R., Dodi, P.L. and Grandi, G. 2010. Canine bladderworm (Capillaria plica) infection associated with glomerular amyloidosis. Vet. Parasitol. 168, 338-341.

Cazelles, C., Bourdeau, P. and Vidal, J. 1989. Capillariose vesicale chez un chien: a propos d'un cas. Point Vet. 21, 41-44.

Davidson, R., Gjerde, B., Vikoren, T., Lillehaug, A. and Handeland, K. 2006. Prevalence of Trichinella larvae and extra intestinal nematodes in Norwegian red foxes (Vulpes vulpes). Vet. Parasitol. 136, 307-316.

Fernández-Aguilar, X., Mattsson, R., Meijer, T., Osterman-Lind, E. and Gavier-Widén, D. 2010. Pearsonema (syn Capillaria) plica associated cystitis in a Fennoscandian arctic fox (Vulpes lagopus): a case report. Acta Vet. Scand. DOI: 10.1186/1751-0147-52-39.

International Renal Interest Society. 2013. http://www. iris-kidney.com [accessed 01 February, 2015].

Kirkpatrick, C.E. and Nelson, G.R. 1987. Ivermectin treatment of urinary capillariasis in a dog. J. Am. Vet. Med. Assoc. 191, 701-702.

Kruger, J.M. and Osborne, C.A. 1993. The role of uropathogens in feline lower urinary tract disease.
Clinical implications. Vet. Clin. North Am. Small Anim. Pract. 23, 101-123.

Magi, M., Guardone, L., Prati, M.C., Mignone, W. and Macchioni, F. 2014. Extraintestinal nematodes of the red fox (Vulpes vulpes) in north-west Italy. J. Helminthol.11, 1-6.

Mariacher, A., Eleni, C., Fico, R., Ciarrocca, E. and Perrucci, S. 2015. Pearsonema plica and Eucoleus böhmi infections and associated lesions in wolves (Canis lupus) from Italy. Helminthologia 52, 364-369.

Maurelli, P.M., Rinaldi, L., Rubino, G., Lia, R., Musella, V. and Cringoli, G. 2014. FLOTAC and mini-FLOTAC for uro-microscopic diagnosis of Capillaria plica (syn. Pearsonema plica) in dogs. BMC Res. Notes 7, 591.

Otranto, D. 2015. Diagnostic challenges and the unwritten stories of $\mathrm{dog}$ and cat parasites. Vet. Parasitol. 212, 54-61. DOI: 10.1016/j. vetpar.2015.06.002.

Rossi, M., Messina, N., Ariti, G., Riggio, F. and Perrucci, S. 2011 Symptomatic Capillaria plica infection in a young European cat. J. Feline Med. Surg.13, 793-795.

Senior, D.F., Solomon, G.B., Goldschmidt, M.H., Joyce, T. and Bovee, K.C. 1980. Capillaria plica infection in dogs. J. Am. Vet. Med. Assoc. 176, 901-905.

Spillmann, S.K. and Glardon, O.J. 1989. Welche diagnose stellen sie? - Welche therapeutischen massnhamen schlagen sie vor? Schweiz. Arch. Tierheilkd. 131, 213-214.

Sréter, T., Széll, Z., Marucci, G., Pozio, E. and Varga, I. 2003. Extraintestinal nematode infections of red foxes (Vulpes vulpes) in Hungary. Vet. Parasitol. 115, 329-334.

Studzińska, M.B., Obara-Gałek, J., DemkowskaKutrzepa, M. and Tomczuk, K. 2015. Diagnosis and therapy of Capillaria plica infection: report and literature review. Acta Parasitol. 60, 563-566.

van Veen, L. 2002. Bladder infection with Capillaria plica in a male dog. Tijdschrift voor diergeneeskunde. 127, 393-394. 